\title{
Familial Robertsonian translocation 15;21 and rare paracentric inv(21): unexpected re-inversion in a child with translocation trisomy 21
}

\author{
Ulrike A Mau' ${ }^{1}$, Uwe R Petruch², Peter Kaiser ${ }^{1}$ and Thomas Eggermann ${ }^{3}$ \\ ${ }^{1}$ Division of Medical Genetics; ${ }^{2}$ Children's Hospital, University of Tübingen; ${ }^{3}$ Institute of Human Genetics, Technical \\ University of Aachen, Germany
}

\begin{abstract}
We present a family with a Robertsonian translocation (RT) 15;21 and an inv(21)(q21.1q22.1) which was ascertained after the birth of a child with Down syndrome. Karyotyping revealed a translocation trisomy 21 in the patient. The mother was a carrier of a paternally inherited RT 15;21. Additionally, she and her mother showed a rare paracentric inversion of chromosome 21 which could not be observed in the Down syndrome patient. Thus, we concluded that the two free chromosomes 21 in the patient were of paternal origin. Remarkably, short tandem repeat (STR) typing revealed that the proband showed one paternal allele but two maternal alleles, indicating a maternal origin of the supernumerary chromosome 21 . Due to the fact that chromosome analysis showed structurally normal chromosomes 21 , a re-inversion of the free maternally inherited chromosome 21 must have occurred. Re-inversion and meiotic segregation error may have been co-incidental but unrelated events. Alternatively, the inversion or RT could have predisposed to maternal non-disjunction. European Journal of Human Genetics (2000) 8, 815-819.
\end{abstract}

Keywords: translocation trisomy 21; paracentric inversion 21q; reinversion

\section{Introduction}

Robertsonian translocations (RT) are the most common balanced structural rearrangements in humans with a frequency in newborns of about 1:1000. ${ }^{1}$ In unbiased studies, $\mathrm{RT}(13 q 14 q)$ and $\mathrm{RT}(14 \mathrm{q} 21 \mathrm{q})$ are the predominant translocations and account for nearly $80 \%$ of all RTs, whilst $\mathrm{RT}(15 \mathrm{q} 21 \mathrm{q})$ is rather uncommon $(0.5 \%)$. The risk of having a chromosomally unbalanced offspring is approximately $17 \%$ in women with RT(13q21q). ${ }^{1}$ Inversions are another group of relatively frequent structural rearrangements. In contrast to the more common pericentric inversions (break and reunion between the short and the long arm), paracentric inversions (PAI) consist of breaks and reunions within the same chromosome arm. In general, PAls are thought to be rare in humans and relatively harmless. ${ }^{1}$ Their frequency in the general population is estimated to be in the range 0.1-0.5:1000 and they are seen in nearly all chromosomes,

Correspondence: Ulrike A Mau MD, Division of Medical Genetics, University of Tübingen, Wilhelmstr. 27, D-72074 Tübingen, Germany. Tel: +49 70712976408; Fax: +49 7071295228;

E-mail: ulrike.mau@uni-tuebingen.de

Received 21 March 2000; revised 19 June 2000; accepted 29 June 2000 most commonly in chromosomes $1,3,5,6,7,11$ and 14 and less frequently in chromosomes 4, 16, 17, 18, 19, 20, 21, 22 and $Y^{2}$. So far, only five cases of PAls of chromosome 21 have been described. Four of them were found in probands with trisomy $21^{2-7}$ (Lindenbaum ${ }^{3}$ and Madan et al, ${ }^{4}$ Niikawa et $\mathrm{al}^{6}$ and Ohta et $\mathrm{al}^{7}$ reported on the same patient respectively). Further testing on additional family members revealed that these inversions were familial and not associated with any symptoms in the healthy carrier. Interestingly, Niikawa's patient with Down Syndrome carried a paracentric inversion in two of his three chromosomes21[47, XY,-21, $+\operatorname{inv}(21)(q 11.2 q 22.13) m a t,+\operatorname{inv}(21)(q 11.2 q 22.13) m a t] .{ }^{6} \mathrm{~A}$ fifth case of PAI of chromosome 21 was detected in amniocentesis; the same PAI was found in the mother. $^{8}$

Here we describe the familial occurrence of both a RT(15q21q) and a PAI of chromosome21 in a healthy woman, whose child carried a translocation trisomy 21 and showed two structurally normal chromosomes 21.

\section{Case report}

The male patient FJ was the second child of healthy Russian parents (mother 25 years, father 28 years, at the child's birth). 
The first child was a healthy girl. No history of miscarriages and no family history of Down syndrome is known. The pregnancy was uneventful and FJ was born after 37 weeks of gestation (birth weight $2340 \mathrm{~g}$, length $46 \mathrm{~cm}$, OFC $31 \mathrm{~cm}$ ). The APGAR score was 8/9/10. The typical features of Down syndrome included (Figure1): upslanted palpebral fissures, Brushfield's spots, epicanthal folds, small nose with low nasal bridge, simian crease of the right hand, small genitals, cryptorchism, large gaps between the first and second toes and a bell-shaped thorax. Marked muscular hypotonia, even for a child with Down syndrome, was noted. Due to muscular hypotonia breathing was difficult and for some days a palate device helped to keep the tongue in place. There were no feeding problems and weight gain was satisfactory. Echocardiography revealed an atrial septal defect (septum secundum) and cardiomegaly, but surgical correction was not then warranted. FJ's developmental progress revealed an overall delay, as he was not able to sit or roll over at one year of age. He is currently receiving physiotherapy and early developmental support.

The family history revealed the following: the mother of FJ has two healthy brothers. The maternal grandparents of FJ had several healthy sisters and brothers, who had healthy children and grandchildren themselves. In both families no previous history of miscarriages or family members with mental or physical retardation was known.

\section{Materials and methods Cytogenetic studies}

Lymphocytes of our proband, his parents and the maternal grandparents were cultured according to standard methods. Chromosome analysis was done by GTG banding. At least 15 metaphase spreads each were analysed at approximately the 400-500 band level.

\section{Molecular genetic studies}

Genomic DNA from peripheral lymphocytes was isolated by a simple salting out procedure. ${ }^{9}$ Delineation of the parental origin of the trisomy 21 was performed by STR typing. The used STRs on chromosome 21 are listed in Table1. Primer and PCR conditions can be obtained from the Genome Database. PCR products were visualised after denaturing gel electrophoresis by silver staining or autoradiograph.

In order to exclude possible mix-up of parental DNA, the samples were tested by PCR for $\mathrm{X}$ - and $\mathrm{Y}$-specific fragments as described by Ellis et al. ${ }^{10}$
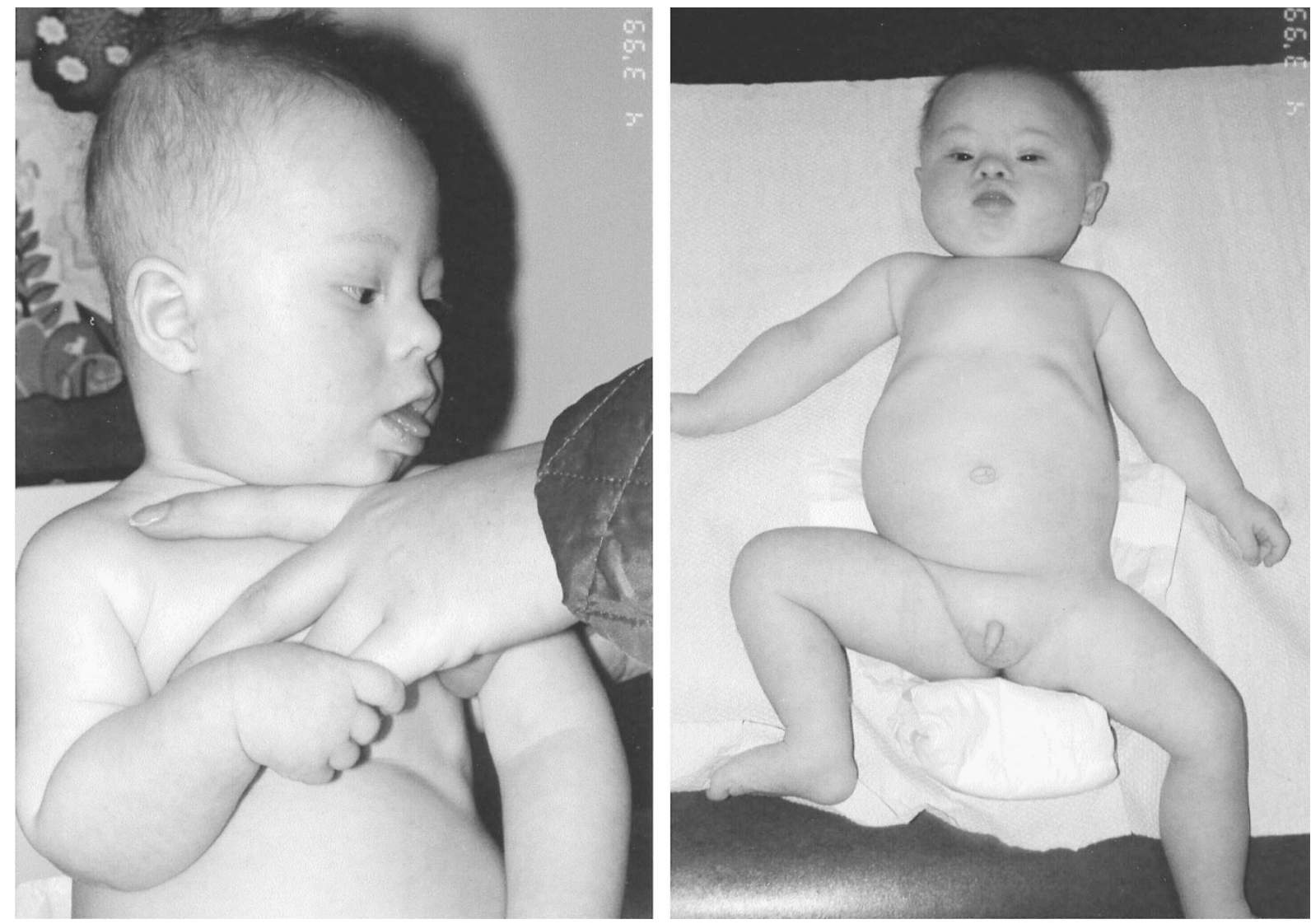

Figure 1 Phenotypic features of our proband at the age of 9 months. a Lateral view showing microcephaly, small nose and protruding tongue. b Frontal view showing dysmorphic stigmata.

\section{European Journal of Human Genetics}


Table 1 Results of chromosome 21 STR typing in the Rob15/21 family. The order of the markers corresponds to the genetic order on chromosome 21 published by Généthon ${ }^{20}$

\begin{tabular}{|c|c|c|c|c|c|c|c|c|}
\hline STR & Het. $^{a}$ & $C M^{a}$ & $\begin{array}{l}\text { Maternal } \\
\text { grandfather }\end{array}$ & $\begin{array}{l}\text { Maternal } \\
\text { grandmother }\end{array}$ & Mother & Father & Patient & Informativity \\
\hline \multicolumn{9}{|l|}{ Cen } \\
\hline D21S1904 & 0.52 & 0.0 & $2-2$ & $1-2$ & $2-2$ & $2-2$ & $2-2$ & - \\
\hline D21S1911 & 0.69 & 2.3 & $1-1$ & $1-1$ & $1-1$ & $1-1$ & $1-1$ & - \\
\hline D21S1256 & 0.65 & 2.3 & $2-2$ & $1-1$ & $1-\overline{2}$ & $3-3$ & $2-2-3$ & Maternal, R \\
\hline D21S265 & 0.84 & 12 & $1-2$ & $2-4$ & $2-2$ & $3-3$ & $2-2-3$ & Maternal \\
\hline D21S1257 & 0.80 & 13 & $1-\overline{3}$ & $2-5$ & $2-3$ & $4-5$ & $3-3-5$ & Maternal, R \\
\hline D21S272 & 0.75 & 13 & $2-3$ & $1-3$ & $2-3$ & $3-4$ & $2-2-3$ & $\mathrm{R}$ \\
\hline D21S269 & 0.72 & 17 & $1-2$ & $1-3$ & $1-3$ & $2-2$ & $1-1-2$ & Maternal, $\mathrm{R}$ \\
\hline D21S1252 & 0.80 & 30 & - & - & $1-1$ & $2-2$ & $1-1-2$ & Maternal \\
\hline D21S270 & 0.85 & 33 & $1-2$ & $2-4$ & $1-4$ & $2-3$ & $1-2-4$ & Maternal, N \\
\hline D21S267 & 0.87 & 33 & $2-4$ & $1-3$ & $2-3$ & $2-4$ & $2-3-4$ & $\mathrm{~N}$ \\
\hline D21S268 & 0.85 & 36 & - & - & 1 & 1 & 1 & U \\
\hline D21S1260 & 0.74 & 44 & $2-4$ & $1-3$ & $1-4$ & $3-3$ & $1-3-4$ & Maternal, N \\
\hline
\end{tabular}

$\mathrm{N}=$ non-reduction of maternal heterozygosity, $\mathrm{R}=$ reduction of maternal heterozygosity, $\mathrm{U}=$ uninformative, $-=$ not typed; ${ }^{\mathrm{H}} \mathrm{Heterozygosity}$ and genetic distance in $\mathrm{CM}$ were obtained from. ${ }^{20}$

\section{Results}

Chromosomal analysis (band level 500) revealed a translocation trisomy 21 in the proband FJ: $46, X Y, \operatorname{der}(15 ; 21)(q 10 ; q 10),+21$ (Figure2). Paternal chromosomes were normal, the maternal karyotype was as follows: $45, X X$, der(15;21)(q10; q10),inv(21)(q21.1q22.1). The Robertsonian translocation was inherited from the maternal grand- father $(45, X Y, \operatorname{der}(15 ; 21)(q 10 ; q 10))$, the grandmother was a carrier of the inv(21q)(46,XX, inv(21)(q21.1q22.1)).

The short tandem repeat (STR) typing results in FJ and his family are listed in Table1. Typing of 21q markers showed one chromosome 21 to be of paternal and two chromosomes 21 to be of maternal origin (Figure2). Furthermore, in distal 21q markers (D21S270, D21S267, D21S1260) maternal

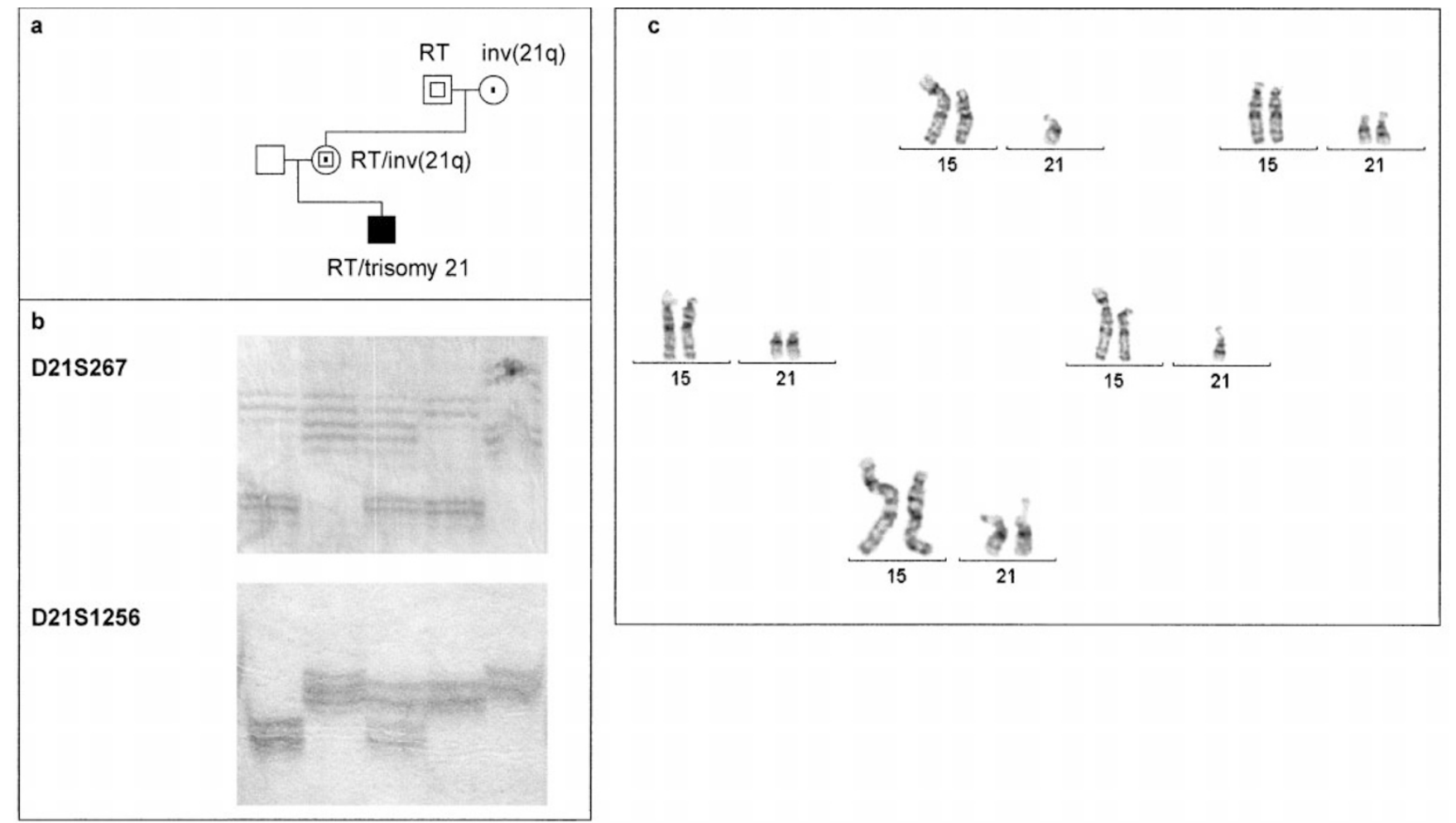

Figure 2 a Pedigree of the RT (15q21q) and inv(21q) family. b Examples of STR typing showing maternal origin and non-reduction/reduction of maternal heterozygosity in the DS patient. c In concordance with the pedigree the partial karyotypes of the family members are presented. 
heterozygosity was maintained in the patient. In contrast, typing of STRs (D21S269-D21S1256) localised in the interstitial segment showed reduction of maternal heterozygosity to homozygosity in the proband. The most proximal markers in $21 q$ were not informative.

\section{Discussion}

In the RT Down syndrome patient described here, the two free chromosomes 21 as well as the translocation chromosome21 appeared to be structurally normal. Therefore we expected at first a paternal origin of the two free chromosomes 21 in spite of the fact that the mother as an RT carrier is at increased risk of having unbalanced offspring. ${ }^{1}$ Furthermore, trisomy 21 cases caused by paternal meiosis errors are rare. ${ }^{11}$ In contrast to our initial expectation, STR typing results indicated that there was paternal contribution of chromosome21, a maternal contribution of the translocated $15 ; 21$, but there was no contribution of the maternally inherited grandmother's PAI21. Instead, the STR pattern indicated that one of the chromosomes 21 was a recombination of the PAI21 and RT21 (Table1).

Since the free chromosome 21 in the proband's mother showed a rare paracentric inversion, it is possible that a re-inversion must have occurred in maternal meiosis followed by non-disjunction (Figure 3 ). This is partially confirmed by the results of STR typing (Table1): distal 21q markers showed non-reduction of maternal heterozygosity in the proband. Thus the two different maternal chromosomes 21 have been inherited, including the one involved in the RT chromosome formation. In maternal meiosis I during homologue pairing, two crossover events may have occurred somewhere in the proximal segment and in the distal segment (proximal to the marker D21S270) outside the inversion, resulting in the structurally normal free chromosome 21 reinversion. The distal recombination was demonstrated in our patient; a proximal crossover was not detectable - unfortunately the respective markers were not informative.

The affected boy FJ had typical signs of Down syndrome showing no unusual symptoms. We suppose that the recombination event between thePAI chromosome and the normal homologue (involved in the RT) led to "balanced reinversion' without any hint of an interstitial duplication or deletion.

Cytologically, the corner-stone of meiotic recombination from a PAl is the 'reverse loop' model. In this model, one crossover event in the inversion loop results in the formation of gametes carrying either a dicentric chromatid, an acentric fragment, a normal chromatid, or a chromatid with an inversion. Gametes containing a dicentric or acentric chromosome are generally perceived as being non-viable. Mar$\operatorname{tin}^{12}$ did not identify any cytogenetically visible meiotic recombinant chromosomes in a PAl carrier. Pettenati and a

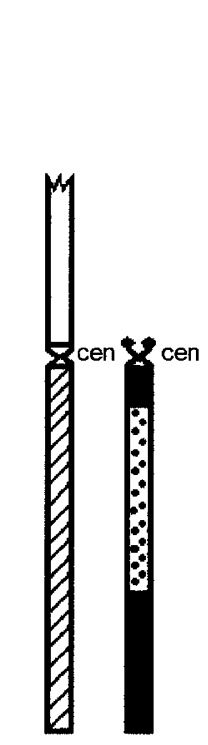

RT

$(15 ; 21) \operatorname{inv}(21)$ b

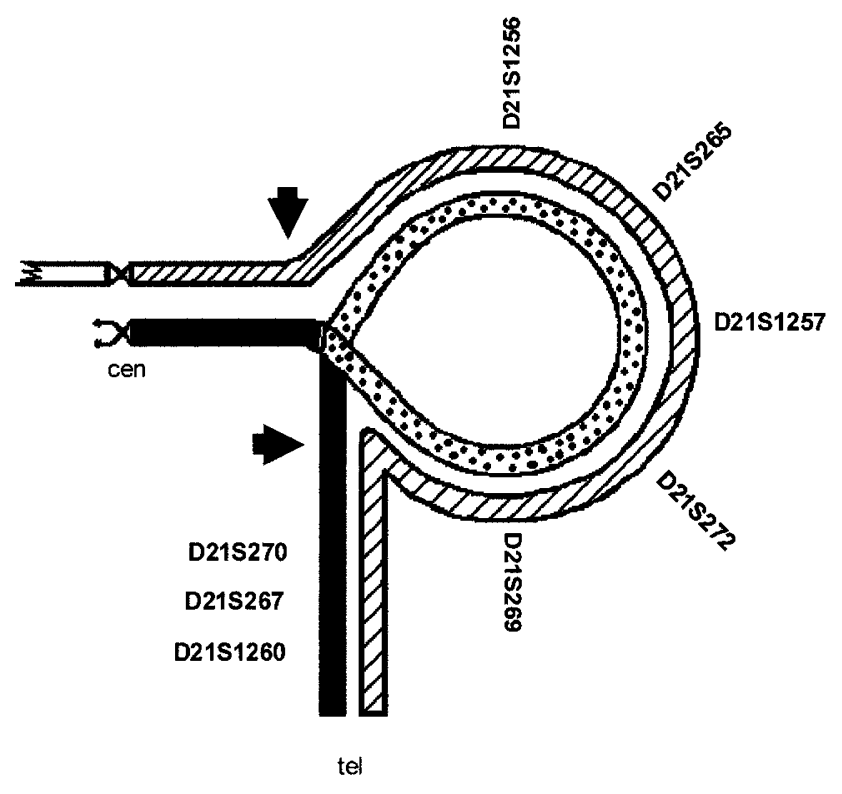

Figure 3 Proposed mechanism of formation of the re-inversion. a Long arms of the RT chromosome21 (hatched parts) and inversion-bearing (blackened/dotted parts) chromosome 21 homologues of the probands mother. b Pairing of the homologues shown in a. The inverted region forms a loop to allow alignment of homologue sequences. Arrows indicate the two potential breakpoints resulting in re-inversion. Informative microsatellites at their putative localisation are presented. c Products of the exchange inherited by the patient. 
co-workers ${ }^{2}$ reviewed 15 PAls with monocentric recombinant chromosomes with duplications and/or deletions. They concluded that the most common viable recombination event from a PAI is either a duplication or a deletion. Nevertheless, the finding of reinversion resulting in a cytogenetically inconspicuous chromosome 21 in our case demonstrates that this segregation product is also detectable.

Familial PAIs are usually considered to bear a relatively small genetic risk: Daniel et $\mathrm{al}^{13}$ did not detect any unbalanced karyotypes in 30 prenatal diagnoses. Pettenati et $\mathrm{al}^{2}$ estimated the rate of viable offspring with recombinant chromosomes to be $3.8 \%$ of the PAI. Neverthel ess, Sutherland et $\mathrm{al}^{14}$ pointed out that several of these alleged monocentric recombinants were original ly reported to arise from parental insertions (3-break rearrangements) and they should not be included in the analysis, therefore decreasing the risk. According to Madan et al carriers of PAIs show no increased incidence of phenotypic abnormalities; ${ }^{4}$ additionally he postulated that the risk of producing abnormal gametes is expected to be low and directly proportional to the length of the inverted segment. ${ }^{15}$ Gardner and Sutherland ${ }^{1}$ commented that virtually all PAI are harmless and that nearly all PAI heterozygotes have been discovered fortuitously and not through an abnormal recombinant attributable to a parental inversion. This corresponds to findings in the family reported here and to the other cases of inv $(21)^{2-7}$ which were detected by chance in Down syndrome patients. However, there have been some reports about PAI carriers who had an offspring with unbalanced karyotypes due to meiotic recombination events. ${ }^{16-18}$ Therefore Yang et al $^{17}$ suppose that the risk for meiotic rearrangements in PAl carriers might be higher than previously expected. Our case illustrates that PAls can lead to monocentric recombinants. We agree with the opinion of Pettenati and Rao ${ }^{19}$ that cytogeneticists, if confronted with PAls, should consider the possibility that alternative mechanisms of pairing and recombination can occur.

A correlation of the coincidental occurrence of the re-inversion of the maternal $\operatorname{inv}(21)$ and the trisomy 21 cannot be excluded in the family reported here. The finding of two different chromosomal aberrations in the same carrier as described here is seldom reported. Therefore, it is difficult to estimate the recurrence risk. Prenatal diagnosis in pregnancies of these carriers should be offered.

\section{Acknowledgements}

We are grateful to the family for participating in this study. We thank U Klein-Vogler for karyotyping and appreciate K Holzmann's helpful suggestions.

\footnotetext{
References

1 Gardner MJ, Sutherland GR: Chromosome Abnormalities and Genetic Counseling. Oxford University Press: Oxford, 1996.
}

2 Pettenati MJ, Rao PN, Phelan MC et al: Paracentric inversions in humans: a review of 446 paracentric inversions with presentation of 120 new cases. Am J Med Genet 1995; 55: 171-187.

3 Lindenbaum RK, Clarke G, Walker J: Down's syndrome and paracentric inversion of 21q. Clin Genet 1981; 20: 377.

4 Madan K, Seabright M, Lindenbaum RH, Bobrow M: Paracentric inversions in man. J Med Genet 1984; 21: 407-412.

5 Rocher E: In: Borgaonkar DS. Repository of Human Chromosomal Variants and Anomalies. Medical Center of Delaware: Newark, Delaware, 1987.

6 Niikawa N, Deng HX, Abe K et al: Possible mapping of the gene for transient myeloproliferative syndrome at 21q11.2. Hum Genet 1991; 87: 561-566.

7 Ohta T, Nakano M, Tsujita T et al: Isolation of a cosmid probe clone corresponding to an inv(21) breakpoint of a patient with transient abnormal myeolopoieses. Am J Hum Genet 1996; 58: 544-550.

8 Torchia BS, Escallon C, Blakemoore KJ, Stetten G: Region-specific FISH probes used to identify and characterize an interstitial paracentric inv(21)(q22.1q22.3). Prenat Diagn 1998; 18: 849-853.

9 Miller SA, Dykes DD, Polesky HF: A simple salting out procedure for extracting DNA from human nucleated cells. Nucleic Acids Res 1988; 16: 1215.

10 Ellis N, Taylor A, Bengtsson BO et al: Population structure of the human pseudoautosomal boundary. Nature 1990; 344: 663-665.

11 Antonarakis SE, Avramoupoulos D, Blouin JL et al: Mitotic errors in somatic cells cause trisomy 21 in about $4.5 \%$ of cases and are not associated with advanced maternal age. Nat Genet 1993; 3: 146-150.

12 Martin RH: Sperm chromosome analysis in a man heterozygous for a paracentric inversion of chromosome7(q11q22). Hum Genet 1986; 73: 97-100.

13 Daniel A, Hook EB, Wulf G: Collaborative USA data on prenatal diagnosis for parental carriers of chromosome rearrangements: risks of unbalanced progeny. In: Daniel A (ed). The Cytogenetics of Mammalian Autosomal Rearrangements, Alan R Liss: New York, 1988; 101-102.

14 Sutherland GR, Callen DF, Gardner RJM: Paracentric inversions do not normally generate monocentric recombinant chromosomes. Am J Med Genet 1995; 59: 390.

15 Madan K: Paracentric inversions: a review. Hum Genet 1995; 96: 503-515.

16 Hoo JJ, Lorenz R, Fischer A, Fuhrmann W: Tiny interstitial duplication of proximal $7 q$ in association with a maternal paracentric inversion. Hum Genet 1982; 62: 113-116.

17 Yang SP, Bidichandani SI, Figuera LE et al: Molecular analysis of deletion (17)(p11.2p11.2) in a family segregating a 17p paracentric inversion: implications for carriers of paracentric inversions. Am J Hum Genet 1997; 60: 1184-1193.

18 Courtens W, Grossman D, Van Roy N et al: Noonan-like phenotype in monozygotic twins with a duplication-deficiency of the long arm of chromosome18 resulting from a maternal paracentric inversion. Hum Genet 1998; 103(4): 497-505.

19 Pettenati MJ, Rao PN: Letter to the editor. Response to Drs Sutherland, Callen, and Gardner. Am J Med Genet 1995; 59: 391-392.

20 Généthon: ftp://ftp.genethon.fr/pub/Gmap/Nature-1995-1995/ data/data_chrom 21 . 